\title{
Diabetes and coronary artery disease: Scary duo of the developing world
}

\author{
Ali Oto
}

1) MD, FESC, FACC, Hacettepe Univ of Medical School, Ankara, Turkey

\section{Summary}

\begin{abstract}
For many years, diabetes mellitus (DM) has been known to be an independent risk factor for atherosclerosis. However in recent years, better understanding of the relations between DM and cardiovasculary system, and especially the accumulating epidemiological evidence caused DM to be referred as "cardivascular disease equivalent" in risk evaluation. Indeed, morbidity and mortality risk related with coronary artery disease in diabetic patients is 2 - 3-fold higher than normal population. Diabetics without a history of previous myocardial infarction and non-diabetics with a history of previous myocardial infarction show similar mortality characteristics. Coronary artery disease is the primary cause of death in adult DM patients. Moreover, when acute coronary syndrome is developed in diabetic patients, both in-hospital and long-term mortality rates are higher than non-diabetics.
\end{abstract}

Keywords: Diabetes mellitus, atherosclerosis, coronary artery disease.

\section{Introduction}

Another important subject is that macrovascular complications induced by diabetes may develop years before emergence of overt diabetes. Epidemiological studies have revealed that the frequency of type $2 \mathrm{di}$ abetes increases with age both in males and females. The incidence of coronary artery disease also increases with age, therefore one should take into account the fact that possibility of co-existence of these two illnesses in elderly would be high. With the aging society, the importance of this duo regarding community health will gradually increase.

While the information regarding coronary artery dis- ease in diabetics has been known for a long time, data regarding the glucometabolic regulation in patients with coronary artery disease started to draw attention rather recently. For example, "Euro-Heart Survey on Diabetes and the Heart" have shown that $20 \%$ of the coronary artery patients have apparent diabetes, $30 \%$ of them have impaired glucose tolerance test, and $5 \%$ of them have impaired fasting glucose. Recently performed GAMI trial showed that the glucose tolerance test performed before discharge of the patients who had acute myocardial infarction, reveals previously unknown glucometabolic disorder in up to $30 \%$ of the patient, and more importantly long-term prognosis is worse in the group with impaired glucose tolerance. 
Diabetes and coronary artery disease: Pathophysiological considerations

There is no doubt that risk factors such as hypertension, dyslipidemia, etc. co-existing with DM have a contribution to the development of atherosclerotic heart disease. However hyperglycemia on its own probably also has an important contribution to pathophysiology. Hyperglycemia results in production of free oxygen radical directly, and indirectly by increasing free fatty acid load. And this is known as the initiator of a process triggering many adverse the metabolic pathway, increasing vascular permeability, decreasing fibrinolytic activity and triggering inflammation.

On the other hand, there is also evidence showing that the activity of renin-angiotensin aldosterone system (RAAS) is increased in diabetic patients. These evidences led RAAS blockers to be used for treatment, and prevention of cardiovascular complications, and thus RAAS became a treatment target. Therefore now in our day, ACE inhibitors and/or angiotension receptor blockers are considered as the essential elements of the current therapy in diabetic patients.

In fact the adverse effects of diabetes on cardiovascular system are much more complicated. In Figure 1, the adverse effects of DM on cardiovascular system are shown. As observed, in addition to hyperglycemia and RAAS activation, there are many negative factors predisposing diabetic patient to the rapid and generalized development of atherosclerosis. New investigations have focused on the pathogenetical relations between diabetes, inflammation, atherosclerosis and atherothrombosis.

In diabetic coronary artery disease patients, multiple vessel disease, left main coronary artery disease,

Figure 1. The adverse effects of DM on cardiovascular system

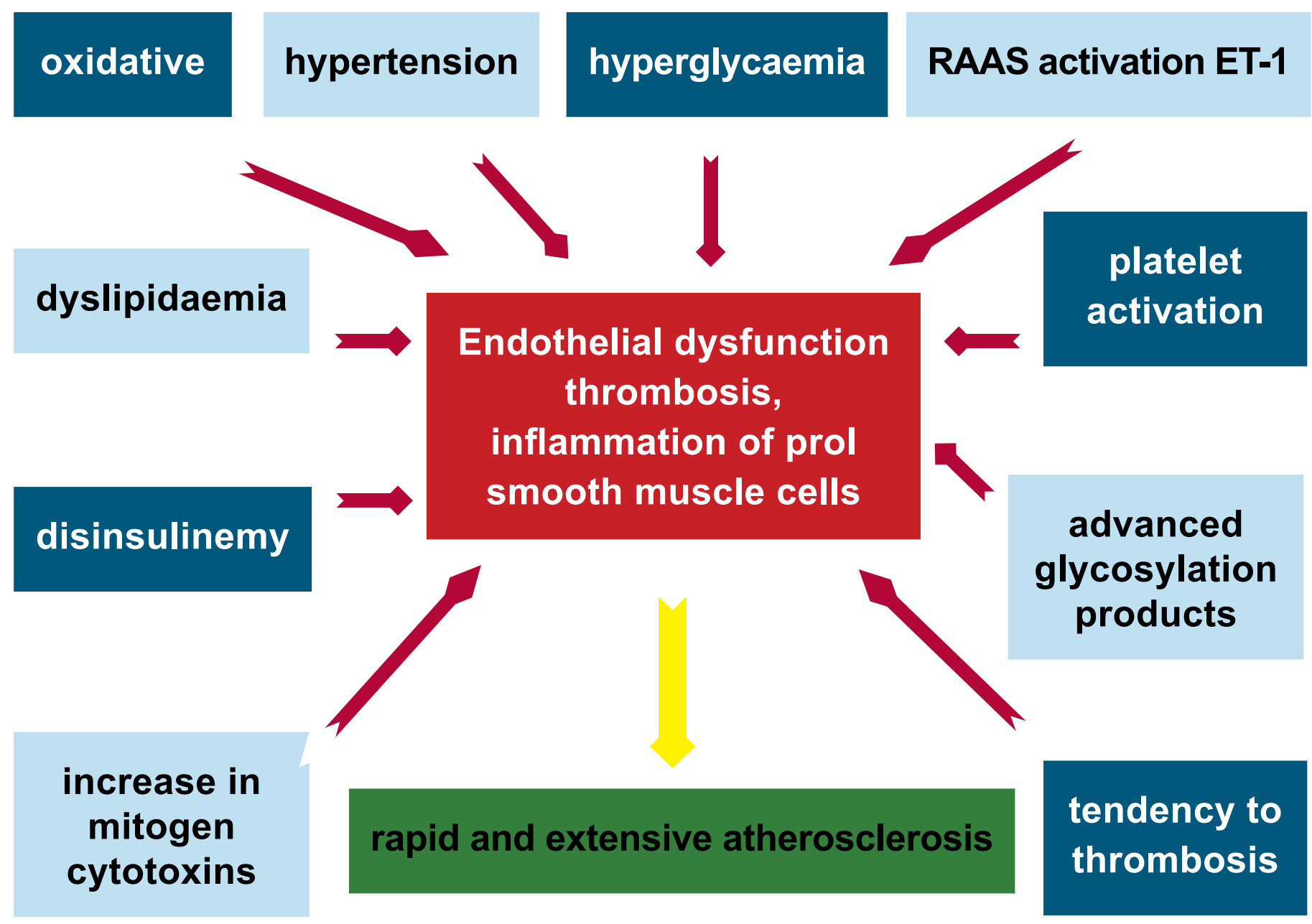


involvement of multiple vessel segments and distal lesions are seen frequently. Furthermore, diabetic atherosclerotic lesions mostly exert unstable plaque characteristis as a result of decreased collagen production, increased degradation, increased matrix metalloprotein activity and increased cytokines.

Glycemic control and coronary artery disease

The glycemia margin for the onset of atherosclerosis and cardiovascular disease in diabetics, has been tried to be explained for many years. Several trials have shown that post-prandial glycemia is an independent risk factor for cardiovascular disease. On the other hand there is evidence showing that the risk of cardiovascular event increases with the elevating fasting blood glucose levels.

While it remains controversial, in light of the available evidence, current opinion is that effective glycemic control is beneficial for prevention of cardivovascular events both type I and type II diabetics. HbAlc is the gold standard method for the monitorization and evaluation of glycemic control. The fact that each $1 \%$ increment in HbA1c may cause up to $20 \%$ increase in the risk of cardiovascular event, is highly significant.
Significant decreases in mortality have been shown after establishing good glycemic control also in patients in intensive care unit due to acute coronary syndrome. The decreased risk of mortality has been associated with the elimination of toxic effects due to acute hyperglycemia which suddenly emerges in critical patients by establishing a good glycemic control, and the direct beneficial effects of insulin treatment in critical patients.

Practical approach recommendations for diabetic patients are as follows:

1. Diabetic patients should be closely monitored and investigated for coronary artery disease.

2. Concomitant risk factors (e.g., hypertension, dyslipidemia) should be detected and corrected.

3. Effective blood glucose control should be established.

4. Antithrombotic medications (aspirin, clopidogrel)

5. RAAS blockers should be given.

Risk assessment should be performed rapidly in diabetic patients having acute coronary syndrome. Fibrinolytic treatment indications in acute myocardial infarction

\section{Diabetes and coronary artery disease: Clinical approach}

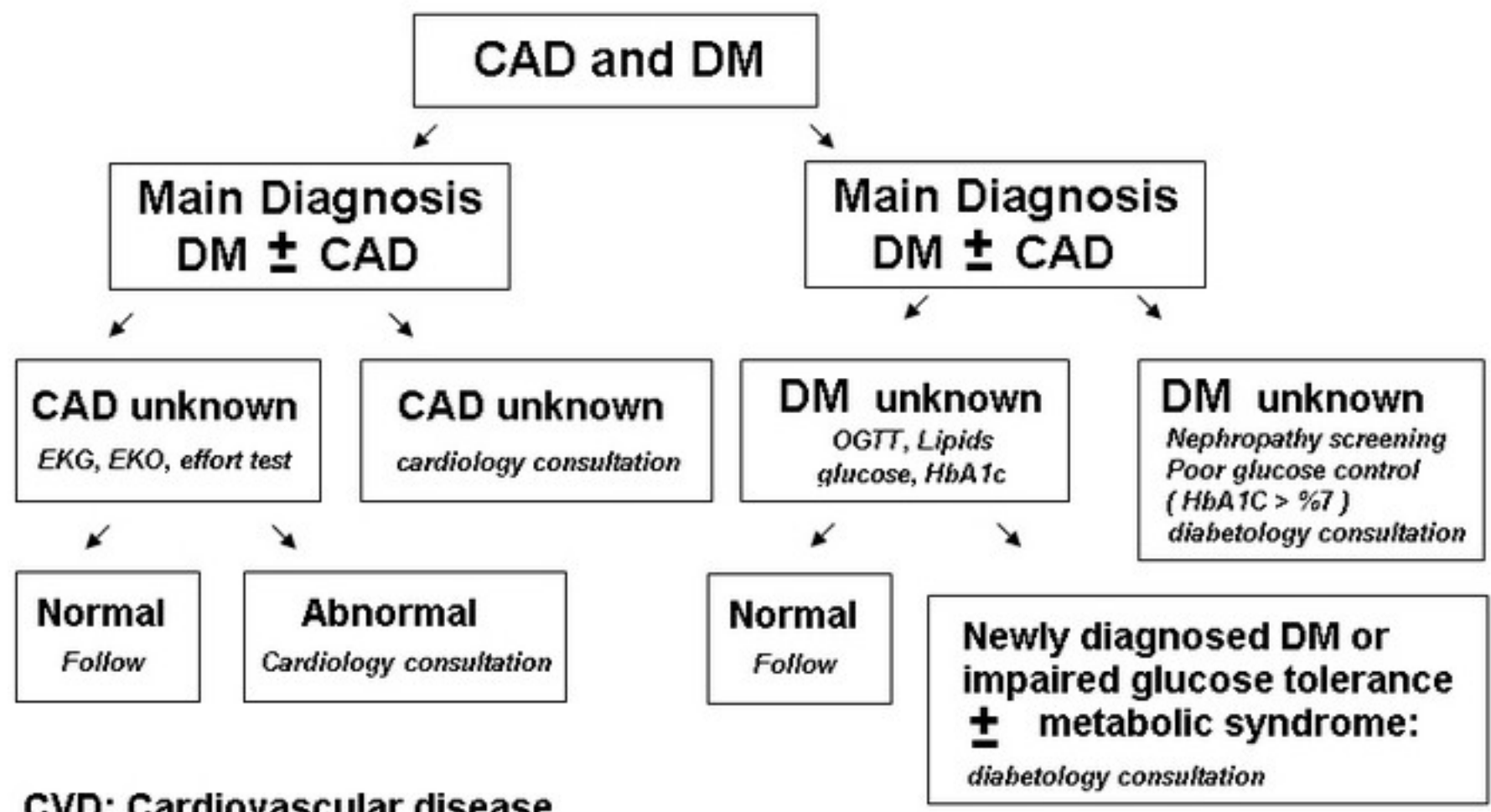


are similar to non-diabetics. If possible, early coronary angiography and mechanical revascularization should be preferred in diabetics. Strict blood glucose control is considered to be beneficial in acute myocardial infarction (AMI). Beta receptor blocking medications decrease morbidity and mortality in diabetics having acute coronary syndrome. Aspirin should be given in doses and indications similar to non-diabetics. However, in diabetics having acute coronary syndrome, addition of clopidogrel to aspirin should be considered. Adding ACE inhibitors to treatment in patients with diabetes and cardiovascular disease, has been shown to reduce cardiovascular events. Treatment targets in patients with diabetes and coronary artery disease are shown in Table I.

It is still controversial to choose coronary bypass or percutaneous coronary intervention in multiple vessel revascularization in diabetic coronary artery patients. Trials featuring surgery, are usually related with pre-stent period. Initial studies performed with stents have shown that while the mortality rates are similar, there are more revascularization procedures involved although less than before in percutaneous coronary intervention. While the use of drug-eluting stents in diabetic patients is still controversial, the tendency is towards using the drug-eluting stents in diabetic patients.

\section{Conclusion}

Diabetes and coronary artery disease are like two sides of the same coin: Today, diabetes is considered to be coronary artery disease equivalent. Many coronary cardiac patients have diabetes. One should expect that the frequency of co-existence of these duo will increase with the aging population.

As the cardiovascular complications of diabetes occur in a very wide glycemia range, prevention, early diagnosis and control of diabetes is highly important for prevention of the development of cardiovascular complications. Therefore, investigating and monitoring the diabetic patients for possible cardiovascular disease is as vitally important as evaluating the coronary cardiac patients for diabetes.
BLOOD PRESSURE $130 / 80 \mathrm{mmHg}$

Renal impairment and $125 / 75 \mathrm{mmHg}$

Proteinuria $>1 \mathrm{~g} / 24 \mathrm{~h}$

\section{STRICT BLOOD GLUCOSE CONTROL}

$\mathrm{HbA} 1 \mathrm{C}<6.5 \% \quad / \quad \mathrm{FBG}<108 \mathrm{mg} / \mathrm{dL}$

Post-prandial $<135 \mathrm{mg} / \mathrm{dL}$ T2 DM

$135-160 \mathrm{mg} / \mathrm{dL}$ T1 DM

LIPID PROFILE $\mathrm{mg} / \mathrm{dL}$

Total cholesterol $<175 \quad / \quad$ LDL-C $<70$

HDL E $>40 \quad / \quad \mathrm{K}>46$

Triglyceride $>150 \quad$ / $\quad \mathrm{TC} / \mathrm{HDL}$

Smoking cessation Mandatory

Regular physical exercise $>30-45 \mathrm{~min} /$ day Weight control

BMI $(\mathrm{kg} / \mathrm{m} 2)<25$

$10 \%$ weight loss if overweight

Waist circumference $<94$ in males $(\mathrm{cm})<80$ in females
Table 1. Recommended treatment targets for patients with diabetes and coronary artery disease

(Modified based on the European Society of Cardiology Cardiovascular Prevention Guidelines) 


\section{References}

1. Ryden L,Standl E, Bartnik M, et.al. Guidelines ondiabetes,pre-diabetes and cardiovascular diseases. Eur Heart J. 2007; 28: 88-136.

2. Graham I, Atar D, Borch-Johnsen K, et al. European Guidelines on cardiovascular disease prevention in clinical practice. European Heart $J$ 2007; 28: 2375-2414

3. Bartnik M,Norhammar A,Ryden L. Hyperglycemia and cardiovascular disease. J Intern Med 2007; 262: 145-56.

4. Stettler C,Alleman S, Jüni P, et al. Glycemic control and macrovascular diseases in type 1 and 2 diabetes mellitus: Meta -analysis of randomized trials. Am Heart J. 2006; 15227-38.

5. Boyden TF,Nallamathu BK,Moscucci M,et al.Meta-analysis of randomized trials of drug eluting stents vs bare metal stents in patients with diabetes mellitus. Am J cardiol. 2007; 99: 1399-1402.

6. Petersen J, Harrington RA. Revascularization of coronary atherosclerosis in patients with diabetes mellitus-There is more to it than meets the image intensifier. Am Heart J. 2005; 149: 190-192.

Received: 05/02/2013

Accepted: 14/05/2013

Published: 15/07/2013

Disclosure and conflicts of interest:

Conflicts of interest were not reported.

\section{Corresponding author:}

Dr. Ali Oto

e-mail: info@alioto.com.tr 